\title{
Radiographic Bone Changes around Immediately Placed Immediately Restored Dental Implants in Periodontally Compromised Sites
}

\author{
Elamrousy WA ${ }^{1 *}$, Nassar $M^{1}$, Ragheb AM$^{2}$, Alnomany FA $^{1}$ and Marzok MA ${ }^{3}$ \\ ${ }^{1}$ Department of Oral Medicine, Periodontology, Oral Diagnosis and Oral Radiology, Faculty of Dentistry, Tanta University, Egypt \\ ${ }^{2}$ Department of Oral Pathology, Faculty of Dentistry, Tanta University, Egypt \\ ${ }^{3}$ Department of Veterinary Surgery, Faculty of Veterinary, Kafrelsheikh University, Egypt
}

\begin{abstract}
Background: The aim of this study is to evaluate radiographic success of immediately restored dental implants placed immediately after extraction of periodontally compromised anterior teeth.
\end{abstract}

Methods: In this prospective clinical trial human study, ten patients were selected from outpatient clinic of the Department of Oral Medicine, Periodontology, Oral Diagnosis, and Radiology, Faculty of Dentistry, Tanta University. Teeth were extracted as a result of sever periodontal destruction, followed by immediate implant placement and immediate implant restoration. Implant survival, bone level was evaluated for each patient at 6, 9 and 12-month follow-up period. Subtraction radiography was performed to compare the baseline image from 12 months follow up period image.

Results: The survival rates of the immediately placed implants in the present study were $100 \%$. Results showed significant reduction of the bone defect depth at 6,9 and 12 months when compared to immediate post-operative record. Also massive increase of the mean bone density scores at 12 months when compared to immediate post operative record.

Conclusion: Based on the results of this clinical trial, placement of implants immediately in fresh extraction sockets affected by periodontal disease followed by immediate restoration may be a valid operative technique that leads to predictable results if adequate preoperative and postoperative care is taken.

Keywords: Bone loss; Dental implants; Immediate loading; Periodontal disease

\section{Introduction}

Today, the placement of implants immediately after tooth extraction has proven to be a predictable treatment strategy with a very high success rate [1]. Several authors [2-5] carried out immediate loading of implants placed in fresh extraction sockets in the anterior (premolar to premolar) region to maintain an excellent soft tissue esthetic profile around the implant-prosthetic restoration with a survival rate of $100 \%$. However, contradictory results were reported by Chaushu et al. [6] with cumulative survival rate of $82.4 \%$ for implants placed in fresh extraction sockets and $100 \%$ for implants placed in healed ridges.

The definition of periodontally compromised/periodontally susceptible patients has been used when evaluating survival and success rates of implants, because periodontal disease has been considered a risk factor for implant therapy. Greater long-term periimplant marginal bone loss versus periodontally healthy subjects, sometimes statistically significant, has been observed in this category of patients $[7,8]$.

Immediately loaded implants present an alternative treatment modality for periodontally compromised patients that might provide a better opportunity to meet patient needs [9]. Still, there are relatively few long-term prospective studies of immediately restored implants and even fewer in periodontally susceptible patients [10,11]. Also, few studies [12-14] have focused on immediate loading of implants placed in fresh extraction sockets, with limited data for soft tissue measurements around implants, the aims of the present clinical study were to evaluate the implant survival and the Peri-implant radiographic changes around immediately provisionalized dental implants placed in fresh extraction sockets in periodontal compromised sites, without GBR procedure.

\section{Materials and Methods}

\section{Patient selection}

Ten patients were selected from outpatient clinic of the Department of Oral Medicine, Periodontology, Oral Diagnosis, and Radiology, Faculty of Dentistry, Tanta University. All patients were provided with written and verbal information about the study and those who fulfilled the criteria were invited to participate in the study. All patients were given informed consent to participate in the study and had the right to withdraw from the study at any time, without consequences to their future care.

Patients were selected according to the following criteria: age ranged from 28 to 37 years of both sexes (four males and six females), adequate bone height apical to the alveolus of the failing tooth $(\geq 5 \mathrm{~mm})$ to ensure primary implant stability, good oral hygiene, completion of skeletal growth, with nil growth considerations affecting implant therapy, psychological acceptance to dental implants and the involved procedures as explained to each patient, and patients should be in apparent good health with no contraindications for surgery, patients with sever periodontitis (chronic or aggressive periodontitis) with anterior periodontally hopeless teeth based on clinical and radiographic assessments. Teeth were characterized as hopeless if they presented with two or more of the following criteria: loss of $75 \%$ of the supporting bone; probing depth (PD) $>8 \mathrm{~mm}$; Class III furcation involvement or grade III mobility; poor crown/root ratios; root proximity; and a history of periodontal abscess.

*Corresponding author: Walid Aly Elamrousy, Department of Oral Medicine, Periodontology, Oral Diagnosis and Oral Radiology, Faculty of Dentistry, Tanta University, Egypt, Tel: +201005724781; E-mail: perioking1@gmail.com

Received July 27, 2013; Accepted October 10, 2013; Published October 12 2013

Citation: Elamrousy WA, Nassar M, Ragheb AM, Alnomany FA and Marzok MA (2013) Radiographic Bone Changes around Immediately Placed Immediately Restored Dental Implants in Periodontally Compromised Sites. Dentistry 3: 161. doi:10.4172/2161-1122.1000161

Copyright: @ 2013 Elamrousy WA, et al. This is an open-access article distributed under the terms of the Creative Commons Attribution License, which permits unrestricted use, distribution, and reproduction in any medium, provided the original author and source are credited. 
Citation: Elamrousy WA, Nassar M, Ragheb AM, Alnomany FA and Marzok MA (2013) Radiographic Bone Changes around Immediately Placed Immediately Restored Dental Implants in Periodontally Compromised Sites. Dentistry 3: 161. doi:10.4172/2161-1122.1000161

Page 2 of 5

Patients were excluded according to the following criteria: the need for prior augmentation of the implant site, presence of persistent and unresolved infection in the implant site, history of smoking, drug or alcohol abuse, poor oral hygiene, presence of systemic conditions that would be a risk factor for the placement of dental implants , severe bruxism or clenching habits and malocclusion, pregnancy, bisphosphonate therapy and history of radiotherapy in the head and neck region for malignancies, chemotherapy for treatment of malignancy.

Patients were scheduled for surgery, tooth extraction, immediate implant placement and immediate implant loading without using the membrane. Surgery included minimal mucoperiosteal flap elevation, tooth extraction, implant placement. At the time of surgery, provisional crowns were fabricated and be seated. 9 months later, permanent restorations were cemented.

All patients received prophylactic antibiotic therapy of $2 \mathrm{~g}$ amoxicillin (or $600 \mathrm{mg}$ clindamycin if allergic to penicillin) 1 hour before the extraction and implant placement procedures, and they continued to take the antibiotic postoperatively, $1 \mathrm{~g}$ amoxicillin (or 300 mg clindamycin) twice daily for 5 days. All patients rinsed for 1 minute with $0.2 \%$ chlorhexidine mouthwash before the surgery (and twice a day for the following 3 weeks) and were treated under local anesthesia using lidocaine with adrenaline at 1:50,000.

All the patients were treated with the same surgical technique consisting of a tooth extraction and simultaneous implant placement. In brief, a full-thickness flap was elevated, and two releasing incisions were performed, extending over the mucogingival junction. Tooth extractions were performed gently to minimize the trauma. After extraction, the socket was carefully curetted, and, subsequently, the implant bed was prepared according to the standard procedure with standard drills following the palatal bony wall as a guide with maximum use of the bone apical to the removed tooth. The longest possible implants were placed with the implant platform placed at the marginal level of the buccal wall. All the implants showed good primary stability (Figure 1).
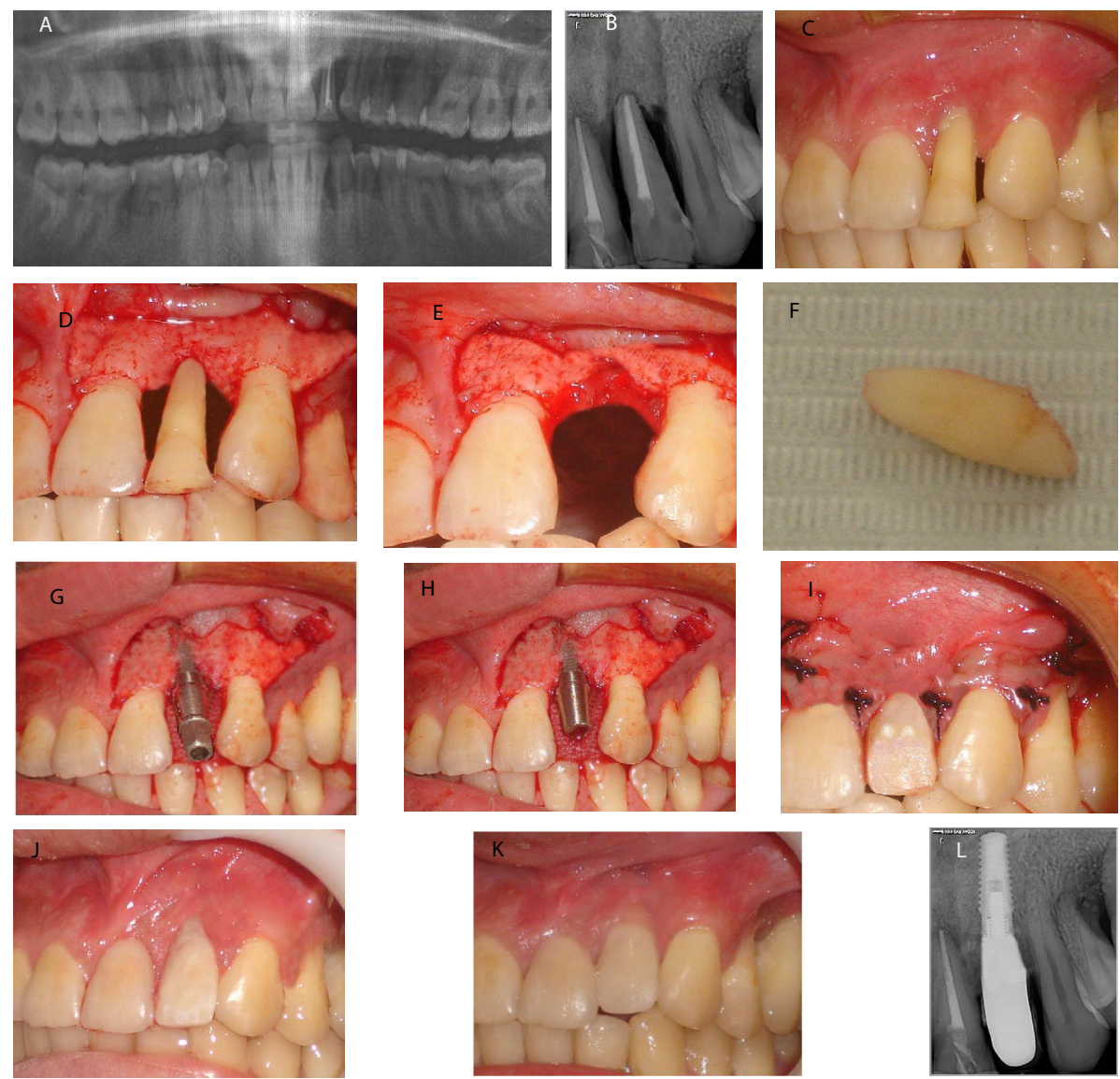

Figure 1: Immediate implants without Duo-Teck membrane

a) Preoperative O.P.G.

b) Preoperative periapical $x$-ray

c) Preoperative study cast.

d) Clear acrylic splint.

e) Preoperative view.

f) Flap reflection

g) Extraction of the involved tooth

h) The extracted tooth.

i) Drilling of the implant bed.

j) Final implant position.

k) Placement of the abutment.

l) Flap closure and temporary crown placement. 
At the time of surgery, provisional crowns were fabricated and seated. 9 months later, permanent restorations were cemented.

Post-operative care: The patients were asked to perform the following measures: cold packs for the first 8 hours, soft diet for the first week, $1 \mathrm{~g}$ amoxicillin (or $300 \mathrm{mg}$ clindamycin) was prescribed 2 times per day for 5 days, ketoprofen $150 \mathrm{mg}$ was prescribed twice per day for 5 days, warm chlorhexidine gluconate $0.1 \%$ mouth wash twice per day in the second post-operative day and was continued for two weeks, avoidance of the surgical site while brushing and eating, the sutures were removed after 7-10 days post-surgically, one week later the operation site was again checked to ensure complete soft tissue healing, and finally and the patients were seen monthly for prophylaxis.

All patients participated in a personally tailored supportive periodontal treatment (follow-up visit at 3 months after treatment) comprising periodontal debridement, root planing at sites with probing depth $>5 \mathrm{~mm}$, and polishing. At these visits, the condition of the soft tissues, the patient's discomfort, and any prosthetic complications were evaluated. The overall level of oral hygiene was also evaluated, and additional instructions were given as needed. Last, once a year, a clinical and radiographic evaluation was performed.

The patients were examined clinically and radiographically at 6,9 and 12 month from implant placement (Figure 2) for the following criteria:

Implant survival: Implant survival was defined in the following
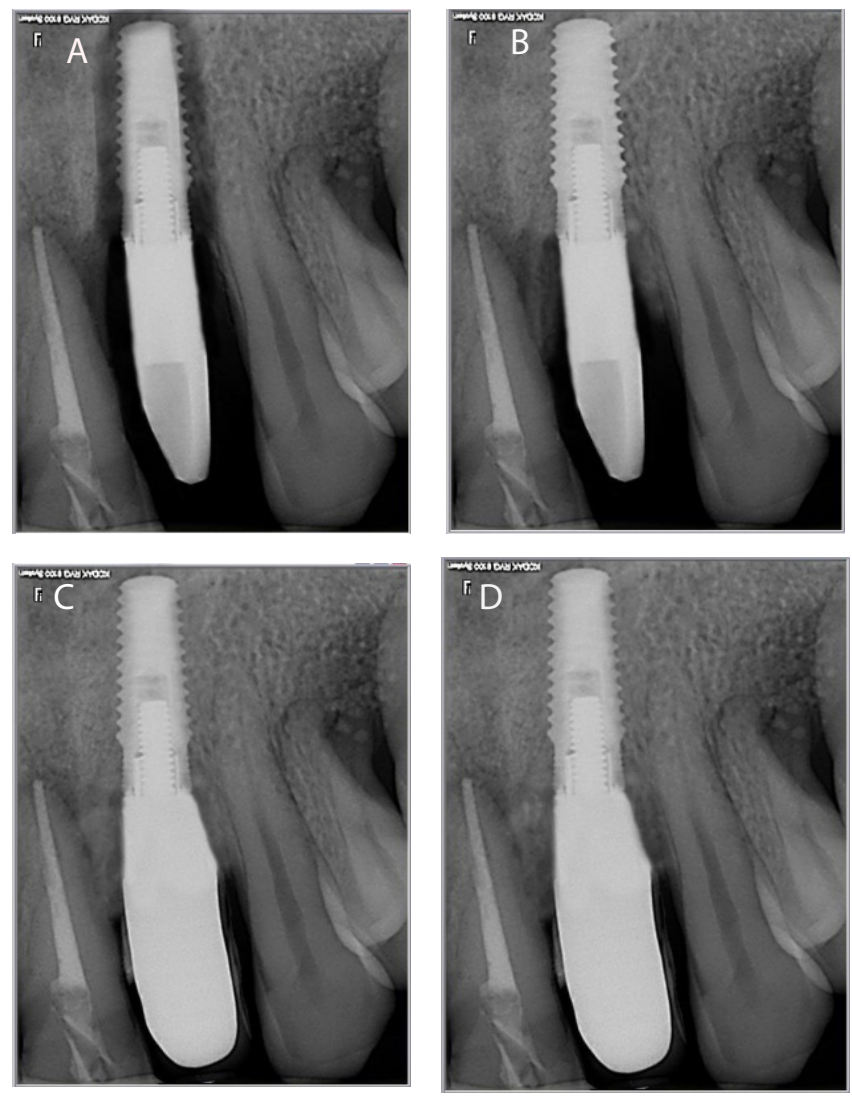

Figure 2: Radiographic evaluation

a) At base line

b) Six months

C) Nine months

d) Twelve months way: the implant was clinically immobile when examined after the crown was clinically removed, there was no pain in the implant area and radiographs did not demonstrate any radiolucency or other pathological conditions adjacent to the implant.

Radiographic evaluation: Standardized periapical radiographs were taken at the time of implant placement, 6, 9 and 12 months after implant placement. The first radiographs after surgery were taken with the implant in situ with temporary restoration, and subsequent radiographs with the restoration were standardized using a radiographic stent.

Marginal bone level: These standardized radiographs were used by Image J software program to calculate:

a) Mesial marginal bone level: the distance from the shoulder of the implant to the first visible bone-to-implant contact (BIC) mesially.

b) Distal marginal bone level: the distance from the shoulder of the implant to the first visible bone-to-implant contact (BIC) distally.

Bone density changes: Subtraction radiography was performed to compare and subtract the baseline image from follow up period images at 12 months. Subtraction procedures were carried out using image analyzer software.

\section{Statistical analyses}

Statistical analyses, including descriptive statistics for all clinical and radiographic parameters, were performed during the entire follow-up period. Implant clinical measurements were calculated by averaging the readings of each implant parameter for each patient, because the within patient variation was much lower than among patient variation. Subsequently, the means and medians were calculated among the means per patient at each study time point. The comparison within the group among the different time points was performed with dependent Student $t$ test (statistically significant at a level of $a=0.05$ ). The $P$ value was set at $<0.05$ with the Bonferroni corrections for multiple comparisons. All the data were analyzed using dedicated statistical software.

\section{Results}

20 patients with anterior periodontally hopeless teeth of the maxillary arch were identified and approached to participate in the study. 10 patients fulfilled the inclusion and exclusion criteria and signed informed consent for participation in the study. The reasons for the 10 excluded patients included: 2 patients due to lack of interest in implant therapy, 3 patients due to inadequate bone volume for implants, one smoker patient, one patient due to malocclusion, 2 patients were out of the age range, and diabetic patient.

The 10 patients participated in this study, consisted of 6 females and 4 males. The mean age of subjects was $30.30 \pm 5.65$, with an age ranging from 22 to 37 years. Six patients had a history of treated periodontitis. All subjects completed the study for the period of data collection.

\section{Location of implants}

A total of 10 implants, 2 dental implants were placed in the upper right central region, 1 in the upper right lateral, 1 in the upper left central, 5 in the upper left lateral and 1 in the upper left canine.

\section{Survival of implants}

The cumulative overall survival rate of all implants placed was $100 \%$. 


\section{Marginal bone level}

It was found that the mean bone defect depth immediately postoperatively was $6.16 \pm 1.52$. The mean bone defect depth dropped to $0.50 \pm 0.26,0.50 \pm 0.27$ and $0.50 \pm 0.26$ at 6,9 and 12 months respectively. Comparing the means of marginal bone defect level throughout the follow up period of group A1 using 2-tail paired samples $\mathrm{t}$-test, it was found that a significant reduction of the marginal bone defect depth at 6,9 and 12 months when compared to immediate post operative record as $t$ values were $13.15,13.14$ and 13.09 respectively $(\mathrm{P}=0.00)$. whereas, the changes in the means of marginal bone defect depth from 6 months to 9 months and from 9 months to 12 months were not statistically significantly different with $t$ values 0.89 and 1.00 respectively ( $\mathrm{P}=0.39$ and 0.34 respectively).

\section{Bone density}

It was found that the bone density after digital subtraction radiography ranged from 103.54 to 123.78 with mean $112.41 \pm 5.81$.

\section{Discussion}

This prospective study describes bone changes occurring up to 1 year after implant placement/implant restoration in periodontally susceptible patients. No implants were lost throughout the study period and the survival rate of dental implants in the present study was $100 \%$. Similar to previous studies of immediately restored dental implants in periodontally treated patients $[15,16]$, the survival rate of dental implants was higher than $90 \%$. These results can be compared to other studies of patients with and without a history of periodontal disease $[15,17,18]$. For example, the results of the present study were comparable to results of immediately restored dental implants in periodontally treated patients obtained by Horwitz et al. [15] with survival rate greater than $90 \%$. In a literature review [16], implant survival rates of immediately loaded implants with immediate or delayed implant placement ranged from $95 \cdot 8 \%$ to $100 \%$. Also, in a previous study Shibly et al. [17] compared bone regeneration and esthetic outcomes between immediate and conventional loading of dental implants placed immediately after extraction in patients with a history of periodontal disease, the implant survival rate at 2 years was $96.7 \%$ and $93.3 \%$ in the immediate loading and conventional loading group respectively. De Boever et al. [18] evaluated the implant survival rate in periodontally non-susceptible patients and in patients with chronic adult periodontitis or with generalized aggressive periodontitis, implant survival in the periodontally non-susceptible patients and chronic adult periodontitis group was $98 \%$ and $96 \%$ after 140 months (showing no difference), but only $80 \%$ after 100 months in the generalized aggressive periodontitis group .

A possible explanation for the high survival rate is the use of stricter patient selection criteria, the inclusion of only patients with a clinically stable periodontal state, an inclusion criterion which was demonstrated by low papilla index and bleeding on probing, and having adequate bone height apical to the alveolus of the periodontally hopeless tooth to ensure primary stability [19]. Karoussis et al. [20] found that periodontal treatment prior to placement of dental implants increases significantly the survival rates of dental implants in periodontally compromised patients as residual periodontal pockets may represent niches of infection for the adjacent implants and periodontal pathogens might be transmitted from teeth to implant. The results are in accordance with Roccuzzo et al. who concluded that periodontally compromised patients who completely adhered to supportive periodontal treatment presented high implant survival rates and enhancement of long-term outcomes of implant therapy [21].
The reported mean bone defect of the present study showed a significant reduction of the bone defect depth at 6,9 and 12 months when compared to immediate post operative record. These results are consistent with several studies [22-29]. Schropp et al. [22] reported that new bone formation occurs in infrabony defects associated with immediately placed implants in extraction sockets. Chen et al. [23] reported that periimplant bone defects following immediate were allowed to heal with a blood clot alone. Defect height reductions between $68 \%$ and $83 \%$ were reported. Covani et al. [24] observed that complete defect fill occurred in the peri-implant gaps following immediate and immediate-delayed implant placement. These observations are corroborated by human histologic studies that have shown spontaneous bone regeneration and osseointegration when peri-implant defects were less than $2 \mathrm{~mm}$ in a horizontal dimension [25]. Results of Spinato et al. [26] suggested that immediate placed and immediate restored single implants are valuable therapeutic options in the maxillary arch and bone graft placement in horizontal gaps have no additional benefit in promoting better clinical outcomes.

In contrast, examining the healing outcomes when the initial periimplant gaps were more than $2 \mathrm{~mm}$, reported that not all sites healed with complete bone fill. Botticelli et al. [27] demonstrated that $25 \%$ of sites with initial orofacial gaps of 2 to $3 \mathrm{~mm}$ healed completely, compared to $78 \%$ of sites with initial gaps of less than $2 \mathrm{~mm}$. Schropp et al. [22] observed that only $52 \%$ of sites with an initial orofacial defect depth of 4 to $5 \mathrm{~mm}$ healed spontaneously in the presence of intact bone walls.

The present study showed that there was a significant and rapid reduction of the mean vertical defect depth in the first three months post-operatively in both groups when compared to that at 6,9 months. This could be explained by Vignoletti et al. [28] who reported that wound healing initiated with a coagulum that was substituted by a provisional matrix at 1 week. Bone formation started concomitant to a marked bone resorption. At 2 weeks, woven bone formation was evident and gradually remodeled into lamellar bone at 4 and 8 weeks.

Evaluation of bone density changes in the jaw bones in the periimplant regions is of interest when studying the healing response after bone grafting procedures. The use of subtraction radiography is not a new concept and has been utilized in dentistry for several decades. It was found that digital subtraction radiography was effective in detecting minimal changes of bone density around dental implants [29].

The present study showed massive increase of the mean bone density scores at 12 months when compared to immediate post operative record, in all groups with no statistical differences between the 4 groups. The results of the present study are supported by Carneiro et al. [30] who assessed longitudinal quantitative changes in bone density around different implant loading protocols and implant surfaces measured by digital subtraction radiography; they concluded that implant loading protocol induced mineral bone gain around singletooth implants after the first year under function.

The results of the present study could be explained by Colombo et al. [31] who found that bone healing in immediate and immediatedelayed placement occurred predominantly on exposed bone surfaces (distance osteogenesis) and not on the implant surface (contact osteogenesis). Also, Vignoletti et al. [28] reported wound healing initiated with a coagulum that was substituted by a provisional matrix at 1 week. Bone formation started concomitant to a marked bone resorption. At 2 weeks, woven bone formation was evident and gradually remodeled into lamellar bone at 4 and 8 weeks. 
Citation: Elamrousy WA, Nassar M, Ragheb AM, Alnomany FA and Marzok MA (2013) Radiographic Bone Changes around Immediately Placed Immediately Restored Dental Implants in Periodontally Compromised Sites. Dentistry 3: 161. doi:10.4172/2161-1122.1000161

The results of the present study could be explained by Degidi and Piattelli [32], Calandriello et al. [33], Romanos et al. [34] and Ghanavati et al. [35] who found that immediately loaded implants' micromovements can improve osseointegration and can dramatically increase the bone density. Also it was shown that immediate loads can increase the mineralization rate in bone-implant interface.

\section{References}

1. Covani U, Marconcini S, Galassini G, Cornelini R, Santini S, et al. (2007) Connective tissue graft used as a biologic barrier to cover an immediate implant. J Periodontol 78: 1644-1649.

2. Covani U, Cornelini R, Barone A (2003) Buccolingual bone remodeling around implants placed into immediate extraction sockets: A case series. J Periodontol 74: 268-273.

3. Cornelini R, Cangini F, Covani U, Wilson TG Jr (2005) Immediate restoration of implants placed into fresh extraction sockets for single-tooth replacement: A prospective clinical study. Int J Periodontics Restortive Dent 25: 439-447.

4. Barone A, Rispoli L, Vozza I, Quaranta A, Covani U (2006) Immediate restoration of single implants placed immediately after tooth extraction. J Periodontol 77: 1914-1920.

5. Kan JYK, Rungcharassaeng K, Lozada J (2003) Immediate placement and provisionalization of maxillary anterior single implants: 1-year prospective study. Int J Oral Maxillofac Implants 18: 31-39.

6. Chaushu G, Chaushu S, Tzohar A, Dayan D (2001) Immediate loading of single-tooth implants: Immediate versus non-immediate implantation. A clinical report. Int J Oral Maxillofac Implants 16: 267-272.

7. Van der Weijden G, Van Bemmen KM, Renvert S (2005) Implant therapy in partially edentulous, periodontally compromised patients: A review. J Clin Periodontol 32: 506-511.

8. Karoussis IK, Kotsovilis S, Fourmousis I (2007) A comprehensive and critical review of dental implant prognosis in periodontally compromised partially edentulous patients. Clin Oral Implants Res 18: 669-679.

9. Horwitz J, Zuabi O, Machtei E (2008) Radiographic changes around immediately restored dental implants in periodontally susceptible patients: 1-year results. Int J Oral Maxillofac Implants 23: 531-538.

10. Horwitz J, Machtei EE (2012) Immediate and delayed restoration of dental implants in patients with a history of periodontitis: a prospective evaluation up to 5 years. Int J Oral Maxillofac Implants 27: 1137-1143.

11. Alves CC, Correia AR, Neves M (2010) Immediate implants and immediate loading in periodontally compromised patients-a 3-year prospective clinical study. Int J Periodontics Restorative Dent 30: 447-455.

12. Anderson E, Haanaes HR, Knutsen BM (2002) Immediate loading of singletooth ITI implants in the anterior maxilla: A prospective 5-year pilot study. Clin Oral Implants Res 13: 281-287.

13. Campelo LD, Camara JRD (2002) Flapless implant surgery: A 10-year clinical retrospective analysis. Int J Oral Maxillofac Implants 17: 271-276.

14. Rocci A, Martignoni M, Gottlow J (2003) Immediate loading in the maxilla using flapless surgery, implants placed in predetermined positions, and prefabricated provisional restorations: A retrospective 3-years clinical study. Clin Implant Dent Relat Res 5: 29-36.

15. Horwitz J, Levin L, Gabay E, Zuabi O, Machtei EE (2012) Immediate restoration of delayed placement of dental implants in patients with treated periodontal disease: 1-year results. Int J Oral Maxillofac Implants 27: 1569-1575.

16. Strub JR, Jurdzik BA, Tuna T (2012) Prognosis of immediately loaded implants and their restorations: a systematic literature review. J Oral Rehabil 39: 704717

17. Shibly O, Patel N, Albandar JM, Kutkut A (2010) Bone regeneration around implants in periodontally compromised patients: a randomized clinical trial of the effect of immediate implant with immediate loading. J Periodontol 81: 17431751.

18. De Boever AL, Quirynen M, Coucke W, Theuniers G, De Boever JA (2009) Clinical and radiographic study of implant treatment outcome in periodontally susceptible and non-susceptiblepatients: a prospective long-term study. Clin Oral Implants Res 20: 1341-1350.
19. Mengel R, Schröder T, Flores-de-Jacoby L (2001) Osseointegrated implants in patients treated for generalized chronic periodontitis and generalized aggressive periodontitis: 3 - and 5-year results of a prospective long-term study. J Periodontol 72: 977-989.

20. Karoussis IK, Salvi GE, Heitz-Mayfield LJ, Brägger U, Hämmerle $\mathrm{CH}$, et al. (2003) Long-term implant prognosis in patients with and without a history of chronic periodontitis: a 10-year prospective cohort study of the ITI Dental Implant System. Clin Oral Implants Res 14: 329-339.

21. Roccuzzo M, DeAngelis N, Bonino L, Aglietta M (2010) Ten-year results of a threearm prospective cohort study on implants in periodontally compromised patients. Part 1: implant loss and radiographic bone loss. Clin Oral Implants Res 21: 490-496.

22. Schropp L, Kostopoulos L, Wenzel A (2003) Bone healing following immediate versus delayed placement of titanium implants into extraction sockets: A prospective clinical study. Int J Oral Maxillofac Implants 18: 189-199.

23. Chen ST, Darby IB, Reynolds EC (2007) A prospective clinical study of nonsubmerged immediate implants: Clinical outcomes and esthetic results. Clin Oral Implants Res 18: 552-562.

24. Covani U, Bortolaia C, Barone A, Sbordone L (2004) Bucco-lingual crestal bone changes after immediate and delayed implant placement. J Periodontol 75 : 1605-1612.

25. Paolantonio M, Dolci M, Scarano A (2001) Immediate implantation in fresh extraction sockets. A controlled clinical and histological study in man. J Periodontol 72: 1560-1571

26. Spinato S, Agnini A, Chiesi M, Agnini AM, Wang HL (2012) Comparison between graft and no-graft in an immediate placed and immediate nonfunctional loaded implant. Implant Dent 21: 97-103.

27. Botticelli D, Berglundh T, Lindhe J (2004) Hard-tissue alterations following immediate implant placement in extraction sites. J Clin Periodontol 31: 820828.

28. Vignoletti $F$, Johansson $C$, Albrektsson $T$, De Sanctis M, San Roman F, et al. (2009) Early healing of implants placed into fresh extraction sockets: an experimental study in the beagle dog. De novobone formation. J Clin Periodontol 36: 265-277.

29. Sakakura CE, Neto RS, Bellucci M, Wenzel A, Scaf G, et al. (2008) Influence of homeopathic treatment with comfrey on bone density around titanium implants: a digital subtractionradiography study in rats. Clin Oral Implants Res 19: 624 628.

30. Carneiro LS, da Cunha HA, Leles CR, Mendonça EF (2012) Digital subtraction radiography evaluation of longitudinal bone density changes around immediate loading implants: a pilot study. Dentomaxillofac Radiol 41 : 241-247.

31. Colombo JS, Satoshi S, Okazaki J, Crean SJ, Sloan AJ, et al. (2012) In vivo monitoring of the bone healing process around different titanium alloy implant surfaces placed into fresh extraction sockets. J Dent 40: 338-346.

32. Degidi M, Piattelli (2003) A Immediately loaded bar-connected implants with an anodized surface inserted in the anterior mandible in a patient treated with diphosphonates for osteoporosis: a case report with a 12-month follow-up. Clin Implant Dent Relat Res 5: 269-272.

33. Calandriello R, Tomatis M, Vallone R, Rangert B, Gottlow J (2003) Immediate occlusal loading of single lower molars using Brånemark System Wide-Platform TiUnite implants: an interim report of a prospective open-ended clinical multicenter study. Clin Implant Dent Relat Res 5: 74-80.

34. Romanos GE, Testori T, Degidi M, Piattelli A (2005) Histologic and histomorphometric findings from retrieved, immediately occlusally loaded implants in humans. J Periodontol 76: 1823-1832.

35. Ghanavati F, Shayegh SS, Rahimi H, Sharifi D, Khalesseh N, et al. (2006) The effects of loading time on osseointegration and new bone formation around dental implants: a histologic and histomorphometric study in dogs. J Periodontol 77: 1701-1707. 